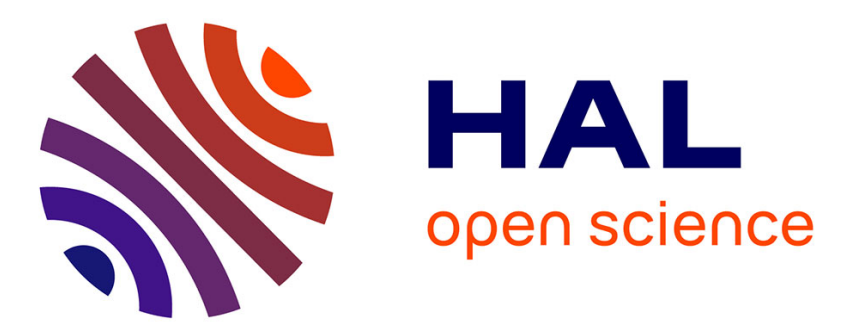

\title{
Electronic and magnetic properties of the multiferroic TbMn2O5
}

\author{
A. Endichi, Hamza Bouhani, H. Zaari, M. Balli, O. Mounkachi, A. El Kenz,
}

A. Benyoussef, Stéphane Mangin

\section{To cite this version:}

A. Endichi, Hamza Bouhani, H. Zaari, M. Balli, O. Mounkachi, et al.. Electronic and magnetic properties of the multiferroic TbMn2O5. Applied physics. A, Materials science \& processing, 2020, 126, 10.1007/s00339-020-03585-4 . hal-02901659

\section{HAL Id: hal-02901659 \\ https://hal.univ-lorraine.fr/hal-02901659}

Submitted on 17 Jul 2020

HAL is a multi-disciplinary open access archive for the deposit and dissemination of scientific research documents, whether they are published or not. The documents may come from teaching and research institutions in France or abroad, or from public or private research centers.
L'archive ouverte pluridisciplinaire HAL, est destinée au dépôt et à la diffusion de documents scientifiques de niveau recherche, publiés ou non, émanant des établissements d'enseignement et de recherche français ou étrangers, des laboratoires publics ou privés. 


\title{
Electronic and magnetic properties of the multiferroic $\mathrm{TbMn}_{2} \mathrm{O}_{5}$
}

\author{
A. Endichi ${ }^{1,2, *}$, H. Bouhani ${ }^{1,2}$, H. Zaari ${ }^{1}$, M. Balli ${ }^{3,4}$, O. Mounkachi ${ }^{1}$, A. El Kenz ${ }^{1}$, A. \\ Benyoussef $^{5,6}$ and S. Mangin ${ }^{2}$ \\ ${ }^{1}$ LaMCScI, B.P. 1014, Faculty of Science-Mohammed V University, Rabat, Morocco \\ ${ }^{2}$ Institut Jean Lamour, UMR CNRS 7198-Université de Lorraine-BP 70239, F-54506 Nancy, \\ France \\ 3 LERMA, ECINE, International University of Rabat, Parc Technopolis, Rocade de Rabat-Salé, \\ 11100, Morocco. \\ ${ }^{4}$ Département de physique \& Institut Quantique, Université de Sherbrooke, J1K 2R1 Québec, \\ Canada \\ ${ }^{5}$ Materials and Nanomaterials Centre, Moroccan Foundation for Advanced Science, Innovation and \\ Reserarch, MAScIR, Rabat, Morocco \\ ${ }^{6}$ Hassan II Academy of Science and Technology, Rabat, Morocco
}

\begin{abstract}
Recently, a reversible and a giant rotating magneto-caloric effect has been pointed out in the multiferroic $\mathrm{TbMn}_{2} \mathrm{O}_{5}$ single crystal, opening the way for new designs of low-temperature magnetic cooling. In this paper, we report a preliminary theoretical work with the aim of enlarging our understanding on the electronic, magnetic and accordingly magnetocaloric features of the $\mathrm{TbMn}_{2} \mathrm{O}_{5}$ compound. Particularly, the $\mathrm{TbMn}_{2} \mathrm{O}_{5}$ magnetic anisotropy is analyzed in terms of X-ray Magnetic Circular Dichroism (XMCD) and X-ray absorption spectroscopy (XAS) spectra.
\end{abstract}

Keywords: Multiferroic materials, Magnetoelectric effect, magnetic anisotropy, XAS, XMCD, DFT.

"Coresponding author : asmae.endi@gmail.com 


\section{Introduction:}

The discovery of new multiferroic compounds exhibiting a strong magneto-electric coupling has aroused great interest since the beginning of the century, justified both by the fundamental issues involved and the prospects for technological applications [1]. The interest of these compounds lies in the coupling between orders, magnetic and electrical, with the possibility, from a static point of view, to manipulate the magnetization by applying an electric field [2]. The more recent discovery of magneto-electric excitations has opened up a new field investigation [3]. In multiferroics, these hybrid excitations, called electromagnons can be understood as magnons excited by the electrical component of a wave electromagnetic and are the signature in the dynamic regime of magneto-electric coupling $[4,5]$. Understanding the mechanisms behind these new excitations is one of the recent challenges of condensed matter physics, and the possibility of modulating these excitations via a field electric and / or magnetic is also an avenue explored for future applications to be defined in the field of information transport, magnetic refrigeration and spintronic devices for example. These materials in which the magnetism and the ferroelectricity are coupled have been widely studied $[6,7,8]$. Studies on $\mathrm{RMn}_{2} \mathrm{O}_{5}$ oxides have shown an important magneto-caloric effect (MEE) that is associated with a unique commensurate-incommensurate magnetic transition [1-9].

In this way, by using relatively low magnetic fields a highly reversible switching of electrical polarization was reported in $\mathrm{TbMn}_{2} \mathrm{O}_{5}$ [10]. On the other hand, it was recently shown that the same compound unveils a giant and reversible rotating magnetocaloric effect (RMCE). Habitually, this compound exhibits an insulating behavior. Its crystalline structure consists of edge-shared $\mathrm{Mn}^{4+} \mathrm{O}_{6}$ octahedra arranged along the c-axis and linked by pairs of $\mathrm{Mn}^{3+} \mathrm{O}_{5}$ pyramids $[11,12,13,14]$. The multiferroic $\mathrm{TbMn}_{2} \mathrm{O}_{5}$ material is characterized also by different exchange interactions involving $\mathrm{Mn}^{4+}, \mathrm{Mn}^{3+}$ and rare earth $\mathrm{R}^{3+}$ ions sublattices, leading to a complex magnetism character. As a result, $\mathrm{TbMn}_{2} \mathrm{O}_{5}$ reveals various magnetic and electric phase transitions $[15,16]$. At $\mathrm{T}_{\mathrm{N}}=43 \mathrm{~K}$, it shows an incommensurate antiferromagnetic (ICM) state with a propagation vector $\mathrm{k}(0.50,0,0.30)$ that transforms into a commensurate antiferromagnetic $(\mathrm{CM})$ phase at $\mathrm{T}_{\mathrm{CM}}=33 \mathrm{~K}$ with $\mathrm{k}=(0.5,0,0.25)$, while a ferroelectric order takes place at $\mathrm{T}_{\mathrm{FE}}=38 \mathrm{~K}[15]$.

Recently, it has been demonstrated that a large thermal effect can be simply produced by rotating the $\mathrm{TbMn}_{2} \mathrm{O}_{5}$ single crystal within the ac-plane in relatively low constant magnetic fields rather than using the conventional magnetization-demagnetization method [1]. Under a constant magnetic field of $2 \mathrm{~T}$, the resulting maximum entropy change from the rotation motion is more than $6 \mathrm{~J} / \mathrm{kg} \mathrm{K}$, while 
the associated adiabatic temperature change is found to exceed $8 \mathrm{~K}$. Such a large RMCE was attributed to different factors such as strong magnetocrystalline anisotropy, low specific heat as well as the enhancement of the magnetization under magnetic fields lower than 3T. In this work, the first principles calculations (DFT) are used to investigate the electronic and magnetic properties of $\mathrm{TbMn}_{2} \mathrm{O}_{5}$, we aim at opening the way for the understanding of the physics behind the RMCE in $\mathrm{TbMn}_{2} \mathrm{O}_{5}$ single crystals and the promising $\mathrm{RMn}_{2} \mathrm{O}_{5}$ family of multiferroics [16].

\section{Computational method}

The $\mathrm{TbMn}_{2} \mathrm{O}_{5}$ compound crystallizes in an orthorhombic symmetry with Pbam space group (number 55). Its lattice parameters are given by: $a=7.2643 \AA, b=8.4768 \AA$ and, $c=5.6700 \AA$ [17]. The electronic and magnetic properties of $\mathrm{TbMn}_{2} \mathrm{O}_{5}$ have been studied using ab-initio calculations with full-potential linearized augmented plane-wave (FP-LAPW) [18] involving the gradient generalized approximation GGA [19], GGA+U and spin-orbit coupling.

Conventional approaches such as the local density approximation (LDA) [20], or that of generalized gradients (GGA) [21], used in density functional theory (DFT), are standards and widely used as an approach to describe the ground state of a large number of insulating, semiconductor and metallic systems. However, it seems to us that they are insufficient to obtain a satisfactory qualitative description of the structure electronics and magnetic properties of correlated systems, so it is necessary to go beyond the DFT to properly treat strong electronic correlations. Several corrections have been developed to provide solutions to this DFT deficiency.

Among those which have been applied, we find the GGA+U method [22, 23], where "U" is the

Hubbard parameter which designates the intra-atomic Coulomb interaction applying to localized orbitals (In general d or f) to correct errors in the DFT.

In $\mathrm{TbMn}_{2} \mathrm{O}_{5}, \mathrm{U}$ is equal to $8 \mathrm{eV}$ for $\mathrm{Tb}$ and $4 \mathrm{eV}$ for $\mathrm{Mn}$ while $\mathrm{J}$ is assumed to be zero for both atoms [19]. The total and partial electron densities are calculated at equilibrium state. The self-consistent calculations are considered to converge with a convergence criterion fixed by a variation of the sixth decimal place in the electronic charge density and with 7x6x9 Monkhorst-Pack mesh implemented in the code WIEN2k [24]. In this work, calculations are performed on the Pbam orthorhombic structure of $\mathrm{TbMn}_{2} \mathrm{O}_{5}$ reported in Figure 1 using the reported crystallographic parameters in ref [17].

To determine the magnetic anisotropy, we have utilized DIPAN program implemented in WIEN2K code [18] that calculates the magnetic dipolar hyperfine field and the dipolar magneto-crystalline anisotropy. 
It is theoretically possible to measure the XMCD signal of any compound having at least one paramagnetic moment and for which the spin-orbit coupling is not zero. The analysis of XMCD spectra allows, with the "sum rules", to go back to the moment of spin and orbit of the studied element $\mathrm{TbMn}_{2} \mathrm{O}_{5}$. It is necessary for that, firstly, to have the absorption field in zero field and calculate it's integral, secondly, to make the difference of the two absorptions obtained for the two polarizations, it is the signal XMCD [24]. This signal is employed to estimate the value of the spin and orbital moments in $\mathrm{TbMn}_{2} \mathrm{O}_{5}$ compound.

Also, the XMCD spin moment sum rule to the $\mathrm{M}_{4,5}$ edges of $\mathrm{Tb}$ is used for the calculation of orbital and spin moments. Considering the transition $3 \mathrm{~d}(\mathrm{c}=2)$ into $4 \mathrm{f}$ final states $(\mathrm{l}=3)$ with the number of holes $\mathrm{n}=41+2-\mathrm{n}_{4 \mathrm{f}}$, the following equations are obtained [25]:

$$
\begin{aligned}
& m_{L}=\left(\mu_{b} / \hbar\right)\langle L z\rangle=-2\left(\left(\int_{M_{4}+M 5} d \omega\left(\mu^{+}-\mu^{-}\right)\right) / \int_{M 4+M 5} d \omega\left(\mu^{+}+\mu^{-}\right)\right) *\left(14-n_{4 f}\right) \\
& m s=\left(\mu_{b} / \hbar\right)\langle S z\rangle= \\
& \left(\left(7 \int_{M 5} d \omega\left(\mu^{+}-\mu^{-}\right)-6 \int_{M 4} d \omega\left(\mu^{+}-\mu^{-}\right)\right) /\left(\int_{M 4+M 5} d \omega\left(\mu^{+}+\mu^{-}\right)\right)^{*}\left(14-n_{4 f}\right)(1+10(\langle T z\rangle /\langle S z\rangle))^{-1}\right.
\end{aligned}
$$

Where the $S z$ and $L z$ are the spin and orbital moment of Tb atoms, respectively.

\section{Results and discussion}

The compound $\mathrm{TbMn}_{2} \mathrm{O}_{5}$ has an orthorhombic structure consisting of octahedra of $\mathrm{Mn}^{4+} \mathrm{O}_{6}$ and bipyramids of $\mathrm{Mn}^{3+} \mathrm{O}_{5}$ linked by their edges and their corners. This structure is illustrated in FIG. 1 . Perfect knowledge of the magnetic orders of the $\mathrm{Mn}^{3+}$ and $\mathrm{Mn}^{4+}$ ions is essential for a good understanding of the multiferroic character of the compound $\mathrm{TbMn}_{2} \mathrm{O}_{5}$.

This material has several magnetic transitions. Below $10 \mathrm{~K}$, the magnetic order of the $\mathrm{Tb}$ ion spins appears and adopts an antiferromagnetic order. Given the complexity of these magnetic orders, we will simplify the diagram of these configurations by looking at the ab plane, in which the ion spins Mn are almost confined.

The front view of the ab plane shows that the crystal structure of $\mathrm{TbMn}_{2} \mathrm{O}_{5}$ is formed by two loops of manganese ions. Each loop consists of a chain of manganese ions $\mathrm{Mn}^{4+}-\mathrm{Mn}^{3+}-\mathrm{Mn}^{3+}--\mathrm{Mn}^{4+}--$ $\mathrm{Mn}^{3+}$ which forms a pentagonal loop. The two loops share two neighboring $\mathrm{Mn}^{3+} \mathrm{O}_{5}$ pyramids. However, if an antiferromagnetic coupling between two neighboring spins is established, it should favor an antiparallel arrangement along the entire chain. However, this is not the case. Indeed, 
geometrically, one cannot form an antiferromagnetic order along a loop formed by five manganese ions. This creates a frustrated magnetic structure which gives rise to more complex magnetic orders.

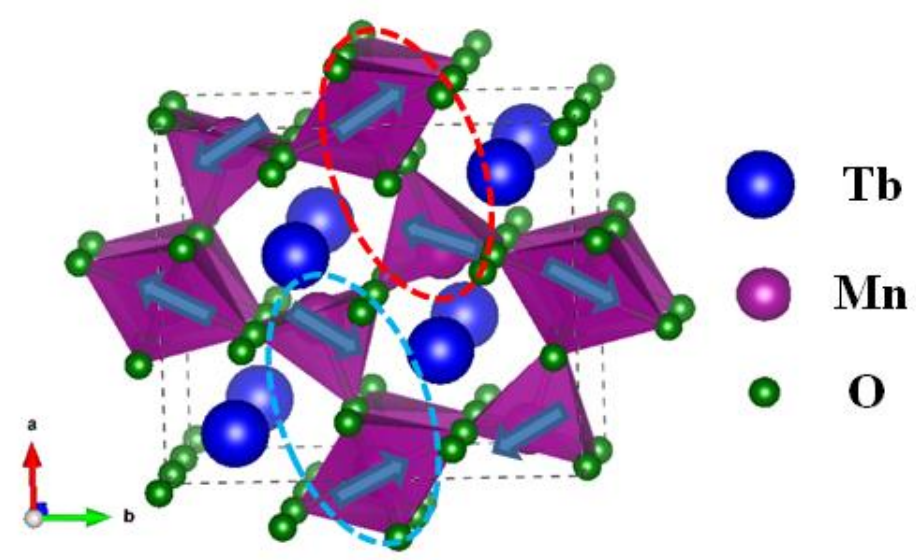

Figure 1: Orthorhombic structure of $\mathrm{TbMn}_{2} \mathrm{O}_{5}$, the blue arrows indicate the magnetic structure of manganese ions.

Figure 1 shows the magnetic configuration of $\mathrm{TbMn}_{2} \mathrm{O}_{5}$ in the commensurable antiferromagnetic phase, the spins of which are ordered antiparallelically along the zigzag chain along the $b$ axis. The neighboring pairs $\mathrm{Mn}^{3+}-\mathrm{Mn}^{4+}$ are alternately coupled in a quasi-ferromagnetic and quasiantiferromagnetic manner along the axis a (ellipses in red and blue dotted lines).

\subsection{Electronic properties}

As we had discussed previously, $\mathrm{TbMn}_{2} \mathrm{O}_{5}$ crystallizes in an orthorhombic structure of space group Pbam, The manganese in this structure have two valences, $\mathrm{Mn}^{3+}$ and $\mathrm{Mn}^{4+}$, corresponding to two crystallographic sites, The cations $\mathrm{Mn}^{4+}$ have an octahedral environment of six oxygen (Fig 2).

The octahedra are joined together by a ridge and form continuous chains along the $\mathrm{c}$ axis. $\mathrm{The}^{\mathrm{Mn}^{3+}}$ environment has pyramids with a square base of five oxygen.

They are organized in dimers in which the two pyramids share an edge. Octahedra and bipyramids constitute the elementary building bricks. Along the $\mathrm{c}$ axis, there is an alternation of planes of pyramids $\mathrm{Mn}^{3+} \mathrm{O}_{5}$, octahedra $\mathrm{Mn}^{4+} \mathrm{O}_{6}$ and rare earth $\mathrm{Tb}^{3+}$.

The crystallographic sites and the coordinates of the ions in the mesh are given in the following table:

\section{Table 1: Atoms positions in $\mathrm{TbMn}_{2} \mathrm{O}_{5}$}




\begin{tabular}{|c|c|c|}
\hline Ion & Name & Position \\
\hline $\mathrm{Tb}^{3+}$ & $\mathrm{Tb}$ & $(\mathrm{x}, \mathrm{y}, 0)$ \\
\hline $\mathrm{Mn}^{3+}$ & $\mathrm{Mn} 1$ & $(0,0.5, \mathrm{z})$ \\
\hline $\mathrm{Mn}^{4+}$ & $\mathrm{Mn} 2$ & $(\mathrm{x}, \mathrm{y}, 0.5)$ \\
\hline $\mathrm{O}$ & $\mathrm{O} 1$ & $(0,0, \mathrm{z})$ \\
\hline $\mathrm{O}$ & $\mathrm{O} 2$ & $(\mathrm{x}, \mathrm{y}, 0)$ \\
\hline $\mathrm{O}$ & $\mathrm{O} 3$ & $(\mathrm{x}, \mathrm{y}, 0.5)$ \\
\hline $\mathrm{O}$ & $\mathrm{O} 4$ & $(\mathrm{x}, \mathrm{y}, \mathrm{z})$ \\
\hline
\end{tabular}
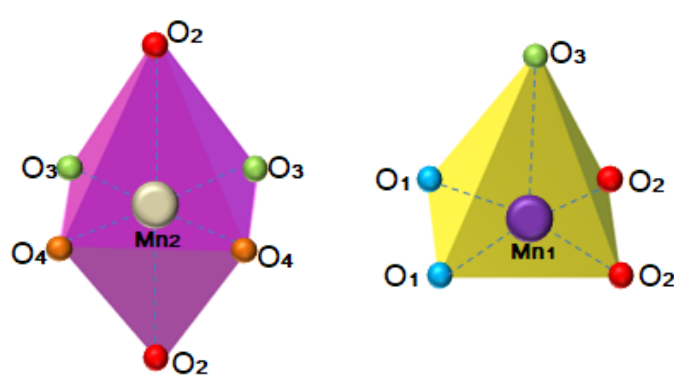

Figure 2: Basic structural units of distorted

$\mathrm{Mn1O}_{5}$ octahedra and $\mathrm{Mn2O}_{6}$ square pyramid

In order to explain the impact of electronic structure on the magnetic properties, we have calculated the total and partial densities of states (PDOS) of $\mathrm{TbMn}_{2} \mathrm{O}_{5}$ with GGA and GGA+U approximations as they are displayed in Fig.3(a) and Fig.3(b). The lower valence bands situated in the range (-7.0 eV) to $(-5.0 \mathrm{eV})$ is due to the Tb-f states. The main contribution to the magnetism comes from $4 \mathrm{f}$ states of $\mathrm{Tb}$ atoms.

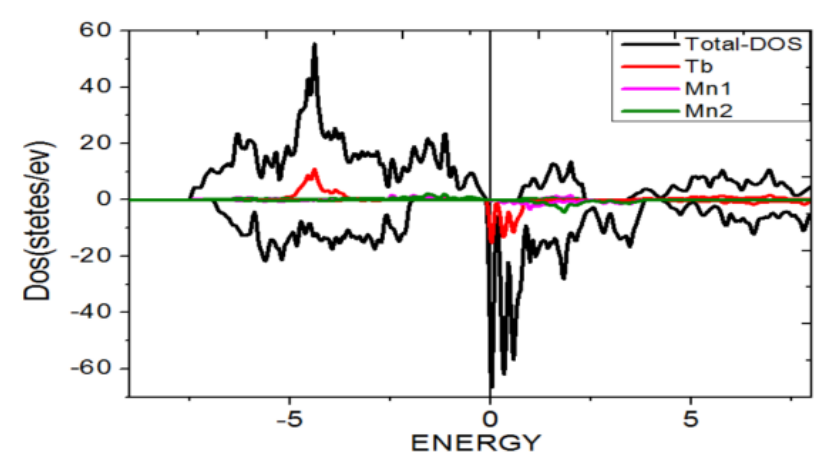

a.

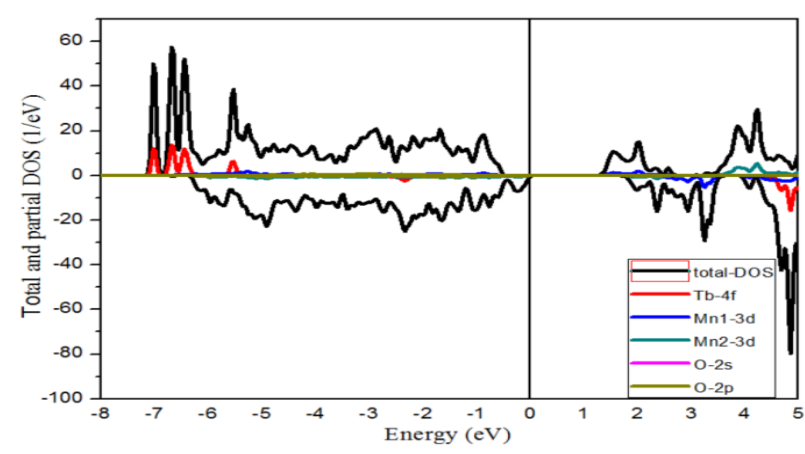

b.

Figure 3: (a) Total density of states for $\mathrm{TbMn}_{2} \mathrm{O}_{5}$ with the GGA approximation. (b) Total and partial density of states for $\mathrm{TbMn}_{2} \mathrm{O}_{5}$ with the GGA+U approximation.

The detail of the structure strongly depends on the nature of Terbium. It is not only the order of moments of $\mathrm{Tb}^{3+}$ which differs from one compound to another of the same family, but also the order of moments of Mn can also be influenced [26, 27]. This indicates the role of $\mathrm{Tb}$ in the magnetic ordering of manganese ions. Indeed, with its strong spin-orbit coupling, the $\mathrm{Tb}^{3+}$ ion has a strong anisotropy. Since that of manganese ions is much weaker, it is expected that the magnetic order in $\mathrm{TbMn}_{2} \mathrm{O}_{5}$ will depend on the anisotropy of Terbium.

The filling of the d orbitals in a pyramidal environment with a square base and in an octahedral environment is done by respecting Hund's rule (the energy of repulsion is minimized by adopting a maximum spin state).

The environment of the terbium, having 8 first neighbors, and the nature of the $4 \mathrm{f}$ orbitals being more complex, the estimation of the degeneration and the filling of these orbitals requires more detailed 
calculations based on a precise crystallographic structure. The nature of the occupied $3 \mathrm{~d}$ and $4 \mathrm{f}$ orbitals and the fine crystallographic structure are essential parameters for the calculation of the exchange integrals.
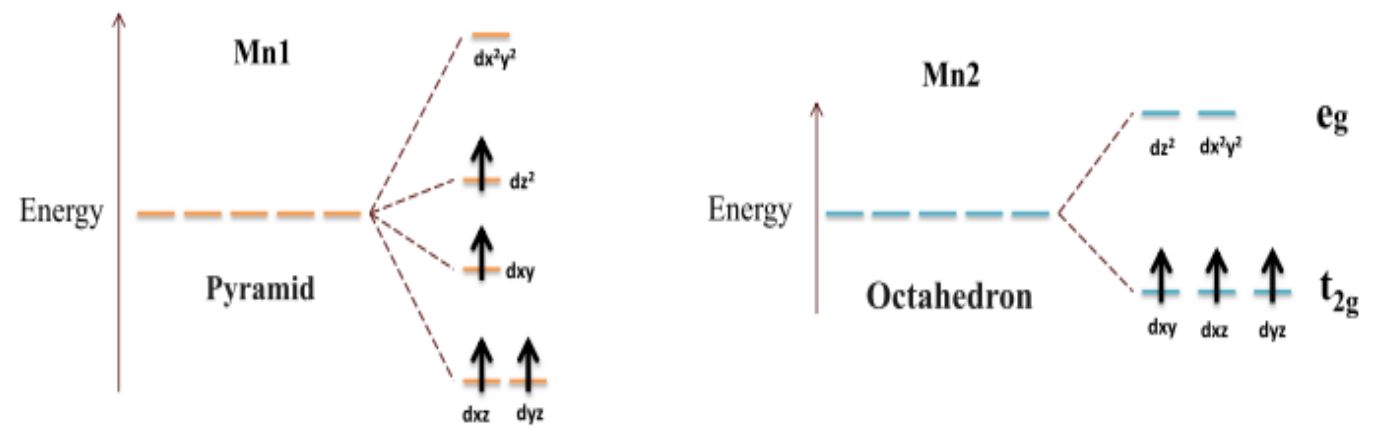

Figure 4: Energy levels and filling of 3d manganese orbitals in a pyramidal (left) and octahedral (right) environment.

The Pbam symmetry of $\mathrm{TbMn}_{2} \mathrm{O}_{5}$ allows a position freedom of $\mathrm{Mn}^{3+}$ ions along the pyramid axis. Such motion alters the Mn-O bonds, and thus the hybridization between the manganese $3 \mathrm{~d}$ and oxygen $2 p$ states. It is worth noting that the $U$ value is taken from ref [28].

The density of states for $\mathrm{Mn} 1$ show that the $\mathrm{t}_{2 \mathrm{~g}}$ level gives rise to two levels: a level which remains stable with a pure metallic character dxy and a level formed by the two hybrid molecular orbitals with a metallic character dxz and dyz, this level is a little destabilized because of the decrease in the binding character metal ligand. The level eg generates an undisturbed level formed predominantly of $\mathrm{dx}^{2}-\mathrm{y}^{2}$, and $\mathrm{a} \mathrm{dz}^{2}$ level which is stabilized (see Fig 4 and 5).

a.

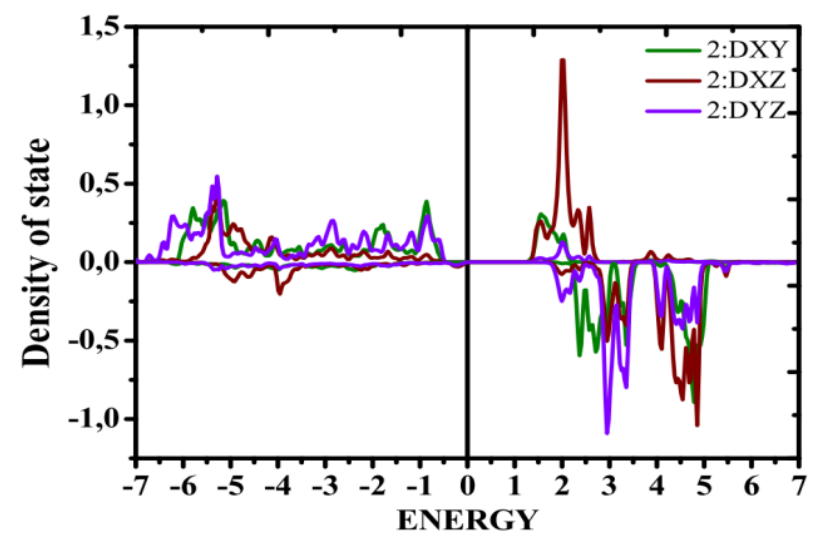

b.

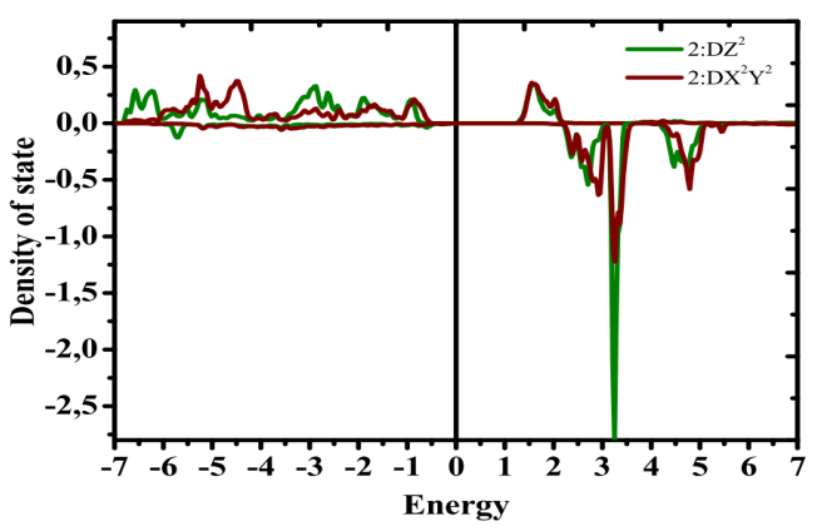


c.

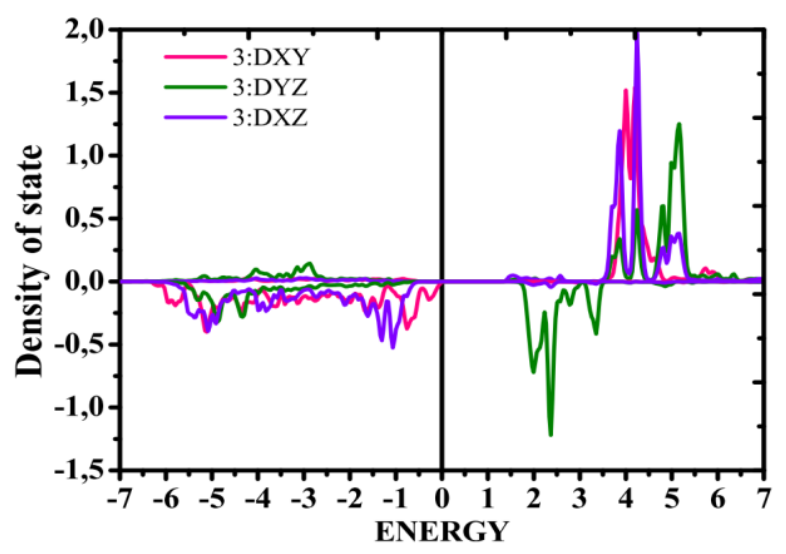

d.

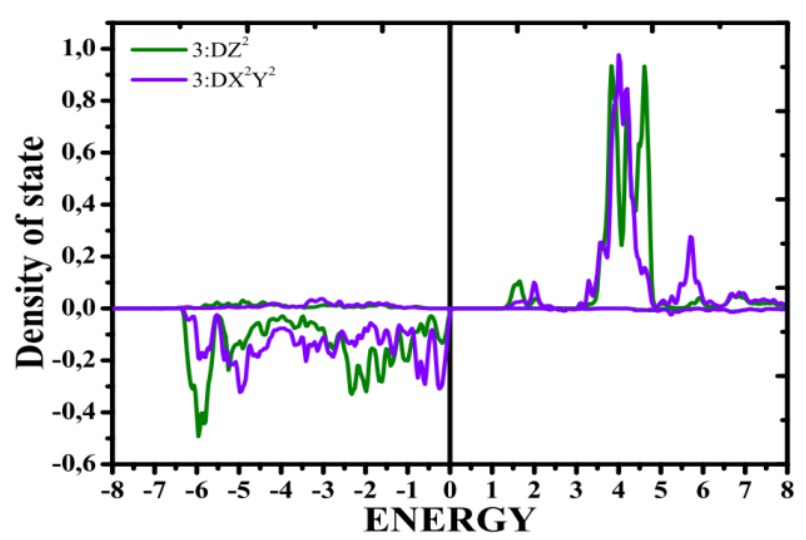

Figure 5: The density of state as a function of energy (eV), of Mn1 (number 2) and Mn2 (number 3) atoms, (a) dxy, dxz and dyz states, (b) $\mathrm{dz}^{2}$ and $\mathrm{dx}^{2} \mathrm{y}^{2}$ states for Mn1 atoms, and (c) dxy, dxz and dyz states, (d) $\mathrm{dz}^{2}$ and $\mathrm{dx}^{2} \mathrm{y}^{2}$ states for Mn2 atoms.

\subsection{Magnetic properties}

In order to well understand the magnetic interactions in the $\mathrm{TbMn}_{2} \mathrm{O}_{5}$ compound, we have calculated the values of $\mathrm{Tb}$ and $\mathrm{Mn}$ spins and their orbital magnetic moments [29]. The sign of the XMCD signal and that of its integral give the relative orientation of the magnetic moments between them and with respect to the applied external field.

For the total magnetic moment given in The Self-Consistent Field (SCF) calculation, it is difficult to distinguish between the contributions from the spins and orbitals since it is equivalent to the difference between up and down spin densities of state.

For these reasons XMCD can gives more details about the value of spin-orbit coupling and calculate separately the spin and orbital moments. The absorption and dichroic spectra XAS and XMCD of $\mathrm{TbMn}_{2} \mathrm{O}_{5}$ are shown in Fig.6(a) and Fig.6(b). At the M edge, the absorption spectrum presents a peak situated at $1208.0 \mathrm{eV}$. At the $\mathrm{L}_{2}$ edge, the spectrum presents one contribution at $1244.5 \mathrm{eV}$. The integral of XMCD and XAS allows the calculation of orbital $\mathrm{m}_{\text {orb }}$ and spin $\mathrm{m}_{\mathrm{s}}$ moments.

a.

b. 

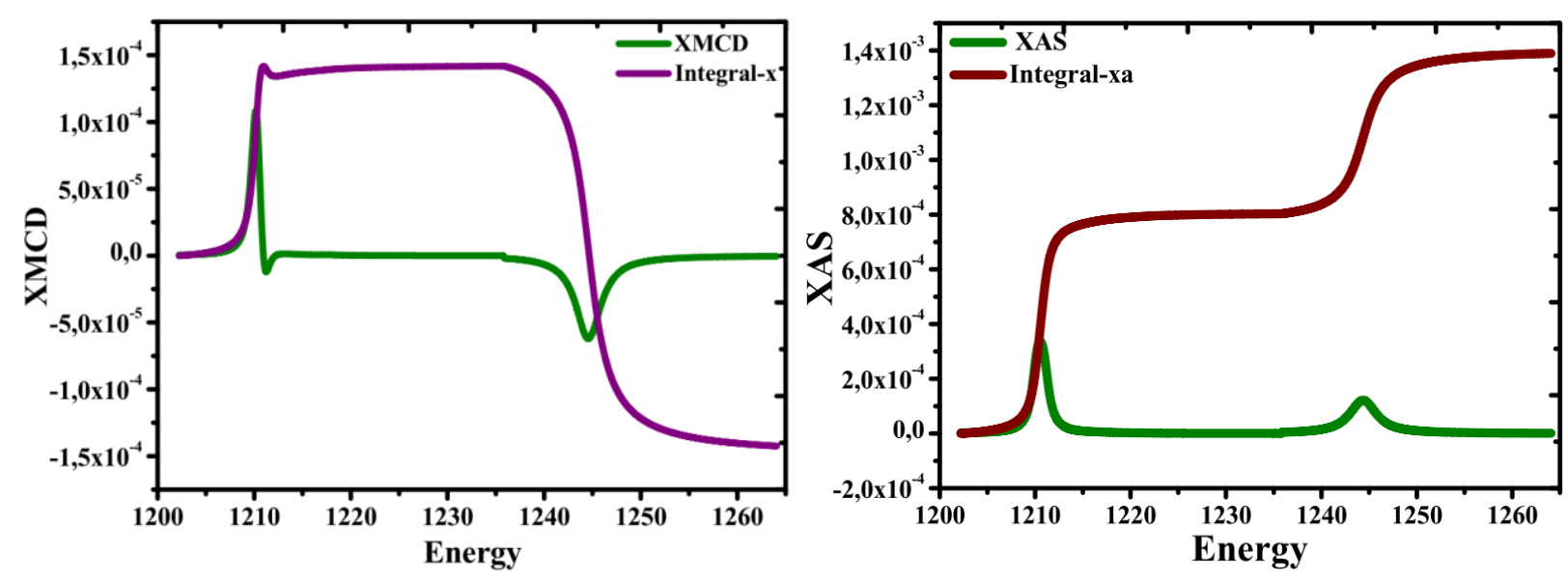

Figure 6: (a) X-ray magnetic circular dichroism (XMCD) spectra of $\mathrm{TbMn}_{2} \mathrm{O}_{5}$. (b) X-ray absorption spectroscopy (XAS) spectra of $\mathrm{TbMn}_{2} \mathrm{O}_{5}$.

The number of $4 \mathrm{f}$-electrons $(3 \mathrm{~d})$ is calculated by integrating the $4 \mathrm{f}$ projected $(3 \mathrm{~d})$ density of states inside each atomic sphere. According to the relations (1) and (2) and using GGA approximation, the values of $\mathrm{m}_{\text {orb }}$ and $\mathrm{m}_{\mathrm{s}}$ are given in Table 2 for both $\mathrm{Tb}$ and $\mathrm{Mn}$ atoms. These values indicate on the strong contribution of $\mathrm{Tb}$ ions to the total magnetic moment [30]. On other hand, the obtained moment for Tb compares well with that experimentally reported in Refs. [1] and [31].

Table 2: The orbital and spin moments of $\mathrm{Tb}, \mathrm{Mn} 1$ and $\mathrm{Mn} 2$ atoms for $\mathrm{TbMn}_{2} \mathrm{O}_{5}$.

\begin{tabular}{|c|c|c|c|c|}
\hline & \multicolumn{3}{|c|}{ XMCD } & Experimental \\
\cline { 2 - 4 } & Tb & Mn1 & Mn2 & Tb \\
\hline Spin moment $(\mu \mathrm{B})$ & 6.9 & -2.01 & 1.44 & - \\
\hline Orbital moment $(\mu \mathrm{B})$ & 1.69 & -0.15 & 0.06 & - \\
\hline Total moment $(\mu \mathrm{B})$ & 8.59 & - & - & $9.34[32]$ \\
\hline
\end{tabular}

In $\mathrm{TbMn}_{2} \mathrm{O}_{5}$ compound, giant magnetocrystalline anisotropy is remarked where the magnetization tends to orient preferentially along the a-axis [1, 31] (along the b-axis for $\mathrm{HoMn}_{2} \mathrm{O}_{5}[33]$ ).As mentioned before, the DFT calculations using xdipan program enabled us to determine the minimal energy corresponding to the easy-axis of magnetization. Thus, the magnetic anisotropy is defined as:

$$
\Delta \mathrm{E}=\mathrm{E}(\text { easy axis) } \mathrm{E} \text { (hard axis). }
$$

The spin-orbit term is included to observe the change in density of states. For $4 \mathrm{f}$ states and $3 \mathrm{~d}$ states, it is known that there is a competition between the spin-orbit effect and the crystal field. The latter is usually strong in the case of $3 \mathrm{~d}$ states (transition metals). Also, the rare earth elements require a spinorbit calculation. So, it is highly important to see the charge distribution in $3 \mathrm{~d}$ and $4 \mathrm{f}$ states in the case of spin orbit coupling (SOC). To understand the anisotropy shown by $\mathrm{TbMn}_{2} \mathrm{O}_{5}$, we calculated the 
energy of the compound following each axis using DIPAN package implemented in WIEN2k program. The calculation consists to compute the total energy for different axes to find the minimal one. For $\mathrm{TbMn}_{2} \mathrm{O}_{5}$, the lower value corresponds to the a-axis, confirming early reported experimental works $[1,34]$.

The Hubbard $\mathrm{U}$ potential makes it possible to localized $\mathrm{f}$ and $\mathrm{d}$ states or the electrons which are delocalized.

The localization of these bands which are responsible for the magnetisms makes it possible to better calculate the magnetic moment and the most stable magnetic phase, as well as the spin orientation of each electron which gives access to the orientation of the magnetization in the compound. In fact we obtain the most probable value of magnetic anisotropy energy (MAE).

Table 3: Magnetic anisotropy of $\mathrm{TbMn}_{2} \mathrm{O}_{5}$ using GGA and GGA+U

\begin{tabular}{|c|c|c|}
\hline \multirow{2}{*}{ Direction } & \multicolumn{2}{|c|}{$\mathbf{E}_{\text {anj }}\left(\mathbf{J} / \mathbf{m}^{3}\right)$} \\
\cline { 2 - 3 } & GGA & GGA+U \\
\hline$[001]$ & $-0,8625.10^{3}$ & $-0,7853.10^{4}$ \\
\hline$[100]$ & $-0,2985.10^{4}$ & $0,1474.10^{5}$ \\
\hline$[010]$ & $-0,7825.10^{3}$ & $-0,6890.10^{4}$ \\
\hline$[110]$ & $-0,1311.10^{4}$ & $0.2292 .10^{4}$ \\
\hline$[101]$ & $-0,4524.10^{3}$ & $0,6133.10^{4}$ \\
\hline$[011]$ & $-0,6167.10^{3}$ & $-0,7190.10^{4}$ \\
\hline$[111]$ & $-0,2786.10^{3}$ & $0.19058 .10^{3}$ \\
\hline
\end{tabular}

It seems from the table 3 that the calculations without applying the potential $U$ give the easy magnetization axis on the a axis while the correction $U$ gave a minimum energy on the [011] axis or the (001) plane.

It should be noted that the magnetic anisotropy is given by the Wien2k code using the Dipan package, which just gives an anisotropy contribution, in particular it is the anisotropy created by the network. For the correction $\mathrm{U}$, the easy magnetization axis is located between the $\mathrm{z}$ axis and the yz plane, which confirms the non-collinearity of the spins of magnetic atoms [35].

\section{Conclusion:}


In summary, our preliminary calculations using the density functional theory study of $\mathrm{TbMn}_{2} \mathrm{O}_{5}$ unveil that it is possible to determine some key parameters such as magnetic moments and electronic structures that are of great interest for the investigation of its entropic behavior (MCE). Particularly, the obtained magnetic moments as well as the anisotropic energies are in fair agreement with previous experimental reports. However, the main challenge remains the simulation of some important magnetothermal parameters of $\mathrm{TbMn}_{2} \mathrm{O}_{5}$ such as specific heat, entropy and adiabatic temperature changes. This point is currently under investigation in our group and any relevant results will be published in a forthcoming publication.

\section{Acknowledgments:}

This work was supported by PHC Toubkal/17/49- 36812NJ project, and by the MESRSFC (Ministere de l'Enseignement Supérieur, de la Recherche Scientifique et de la formation des cadres) in the framework of the national program PPR under contract no. PPR/2015/57.

\section{References:}


[1] M. Balli, S. Jandl, P. Fournier, and D. Z. Dimitrov, Appl. Phys. Lett 108, 102401 (2016).

[2] H. Katsura, N. Nagaosa, and A. V. Balatsky, Phys. Rev. Lett. 95, 057205 (2005).

[3] M. Fiebig, J. Phys. D: Appl. Phys. 38, R123 (2005).

[4] Graeme Eoin Johnstone, "Neutron and X-ray Scattering Study of Magnetic Manganites", University of Oxford, 2012.

[5] S.-W. Cheong and M. Mostovoy, Nature Materials 6, 13 (2007).

[6] A. B. Sushkov, R. V. Aguilar, S. Park, S-W, Cheong and H. D. Drew, Phys Rev Lett. 98, 027202 (2007).

[7] J. Varignon, S. Petit, A. Gellé, and M. B. Lepetit, “J Phys Condens Matter.;25(49):496004”, 2013.

[8] L. C. Chapon, P. G. Radaelli, G. R. Blake, S. Park, and S.-W. Cheong, Phys. Rev. Lett. 96, 097601 (2006).

[9] A. RAMIREZ, Geometrical Frustration, chapter 4, p. 423, Elsevier Science B.V., 2001.

[10] N. Hur, S. Park, P.A. Sharma, J. S. Ahn, S. Guha and S-W.Cheong, Nature 429, 392 (2004).

[11] G. R. Blake, L. C. Chapon, P. G. Radaelli, S. Park, N. Hur, S-W. Cheong, and J. RodríguezCarvajal, Phys. Rev. B 71, 214402 (2005).

[12] Tay-Rong Chang, Horng-Tay Jeng, Chung-Yuan Ren, and Chen-Shiung Hsue, Phys. Rev. B 84, $024421(2011)$.

[13] N. Hur, S. Park, P. A. Sharma, S. Guha, and S-W. Cheong, Phys. Rev.Lett. 93, 107207 (2004).

[14] D. Tzankov, V. Skumryev, M. Aroyo, R. Puzniak, M.D. Kuz'min, M. Mikhov, Solid.Stat. Comm. 147, 212 (2008).

[15] L. C. Chapon, G. R. Blake, M. J. Gutmann, S. Park, N. Hur, P. G. Radaelli, and S. W. Cheong, Phys. Rev. Lett.93, 177402 (2004).

[16] I. A. Sergienko and E. Dagotto, Phys. Rev. B. 73, 094434 (2006).

[17] A. Muñoz, J. A. Alonso, M. T. Casais, M. J. Martínez-Lope, J. L. Martínez, and M. T. FernándezDíaz, Phys. Rev. B 65, (2002)144423

[18] P. Blaha, K. Schwarz, G. Madsen, D. Kvasnicka, and J. Luitz, WIEN 2k, Augmented Plane Wave Local Orbitals Program for Calculating Crystal Properties, Vienna, Austria, 2001.

[19] J. P. Perdew, K. Burke, and M. Ernzerhof, Phys. Rev. Lett. 77, 3865 (1996)

[20] J. P. Perdew, K. Burke, M. Ernzerhof, Generalized Gradient Approximation Made Simple, Phys. Rev. Lett. , Vol. 77, No. 18, (1996).

[21] L. Hedin, B. I. Lundqvist, Explicit local exchange-correlation potentials, J. Phys. C: Solid St. Phys. , Vol. 4, (1971).

[22] S.Y. Savrasov and G. Kotliar. Phys. Rev. Lett., 84, 3670 (2000).

[23] B.-T. Wang, H. Shi, W. Li, and P. Zhang, Phys. Rev. B 81, 045119 (2010). 
[24] H. J. Monkhorst and J. D. Pack, Phys. Rev. B 13, 5188 (1976).

[25] P. Carra, B. T. Thole, M. Altarelli and X. Wang, Phys. Rev. Lett., 70, 694, Feb. 1993.

[26] L. C. Chapon, G. R. Blake, M. J. Gutmann, S. Park, N. Hur, P. G. Radaelli, and S.-W. Cheong, "Structural anomalies and multiferroic behavior in magnetically frustrated $\mathrm{TbMn}_{2} \mathrm{O}_{5}$," Phys. Rev. Lett., vol. 93, p. 177402, Oct 2004.

[27] P. G. Radaelli, C. Vecchini, L. C. Chapon, P. J. Brown, S. Park, and S.-W. Cheong, "Incommensurate magnetic structure of $\mathrm{YMn}_{2} \mathrm{O}_{5}$ : A stringent test of the multiferroic mechanism," Physical Review B, vol. 79, p. 020404, jan 2009.

[28] C. Tay-Rong, J.Horng-Tay, R. Chung-Yuan, H. Chen-Shiung, Phys Rev B,84.024421(2011).

[29] B. T. Thole, P. Carra, F. Sette and G. van der Laan, Phys. Rev. Lett., 68, 1943 (1992).

[30] C. Wilkinson, P. J. Brown, and T. Chatterji, Phys. Rev. B. 84, 224422 (2011).

[31] M. Balli, S. Jandl, P. Fournier, D. Z. Dimitrov, Crystals 2017, 7, 44.

[32] J. Jensen and A. R. Mackintosh, Rare Earth Magnetism (Oxford Univ. Press, Oxford, 1991)

[33] H. Bouhani, A. ENDICHI et al, Journal of "Materials Chemistry and Physics", 10.1016/ 2019.04.044.

[34] Chenjie Wang, Guang-Can Guo, and Lixin He, Materials Science, 2007, arXiv:0711.2539v1.

[35] M. Balli, B. Roberge, P. Fournier, et S. Jandl, « Review of the Magnetocaloric Effect in $\mathrm{RMnO}_{3}$ and $\mathrm{RMn}_{2} \mathrm{O}_{5}$ Multiferroic Crystals », Crystals, vol. 7, $\mathrm{n}^{\mathrm{o}}$ 2, p. 44, févr. 2017, doi: $10.3390 /$ cryst 7020044 . 